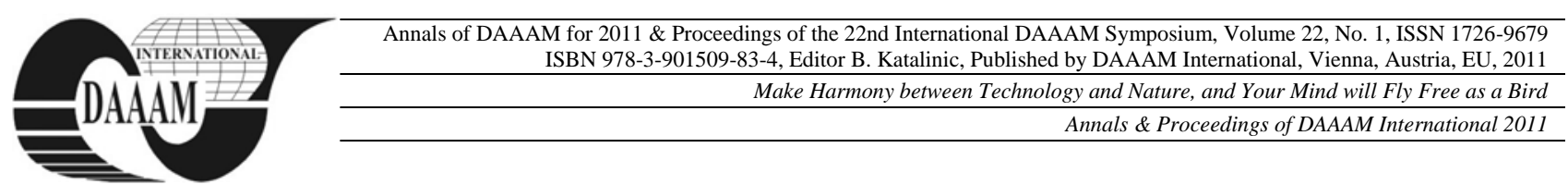

\title{
NYQUIST CRITERION FOR SYSTEMS WITH DISTRIBUTED DELAYS
}

\section{PEKAR, L[ibor]}

\begin{abstract}
Asymptotic and strong stability, spectrum analysis and stabilization of linear time-invariant time delay systems have been challenging tasks in control theory during last decades. The aim of this contribution is to derive and present closed loop stability criterion for systems with distributed delays based on the argument principle. Both retarded and neutral delayed systems are considered to obtain general results. Proofs of statements and theorem and demonstrative examples are omitted due to the limited space.
\end{abstract}

Key words: time delay systems, argument principle, distributed delays, Nyquist criterion

\section{INTRODUCTION}

Delay in the feedback for linear time-invariant time delay systems (LTI-TDS) can significantly deteriorate the quality of control performance, namely stability and periodicity (Stépán, 1989). For lumped delays, the denominator quasipolynomial decides about the control system asymptotic stability since its zeros agree with system poles with the same meaning as for polynomials; however, the spectrum is infinite due to a quasipolynomial transcendental form. Dealing with distributed delays (either in state or input variables) is a rather more involved since some roots of transfer function numerator and denominator coincide and thus some denominator zeros are not the system poles. Moreover, stability of neutral systems can not be sufficiently studied only in terms of asymptotic stability because of the fact that neutral LTI-TDS can be destabilized by even infinitesimally small changes in delays. This brought the concept of so called strong stability (Hale \& Verduyn Lunel, 1993) closely related to notion of formal stability (Pontryagin, 1942).

In this contribution, we derive the generalized Nyquist criterion for LTI-TDS with distributed delays, for both retarded and neutral cases, ensuring the closed loop asymptotic and robust stability. The findings in papers mentioned above were obtained via the argument principle, or equivalently, via the Mikhaylov stability criterion (Górecki et al., 1989; Zítek \& Vyhlídal, 2008). The paper extends results obtained for retarded systems with lumped delays only in (Pekař et al., 2011).

\section{PRELIMINARIES}

A state-space description of a LTI-TDS can be provided by the set of FDEs in the form (1) where $\mathbf{x} \in \square^{n}$ is a vector of state variables, $\mathbf{u} \in \square^{m}$ stands for a vector of inputs, $\mathbf{y} \in \square^{l}$ represents a vector of outputs, $\mathbf{A}_{i}, \mathbf{A}(\tau), \mathbf{B}_{i}, \mathbf{B}(\tau), \mathbf{C}, \mathbf{H}_{i}$ are real matrices of compatible dimensions, sums with $0 \leq \eta_{i} \leq L$ stand for lumped (point-wise) delays and convolution integrals express distributed delays. If $\mathbf{H}_{i} \neq \mathbf{0}$ for any $i=1,2, \ldots N_{H}$, model (1) is called neutral; on the other hand, if $\mathbf{H}_{i}=\mathbf{0}$ for every $i=$ $1,2, \ldots N_{H}$, so called retarded LTI-TDS is obtained.

$$
\begin{aligned}
\frac{\mathrm{d} \mathbf{x}(t)}{\mathrm{d} t}= & \sum_{i=1}^{N_{H}} \mathbf{H}_{i} \frac{\mathrm{d} \mathbf{x}\left(t-\eta_{i}\right)}{\mathrm{d} t}+\mathbf{A}_{0} \mathbf{x}(t)+\sum_{i=1}^{N_{A}} \mathbf{A}_{i} \mathbf{x}\left(t-\eta_{i}\right) \\
& +\mathbf{B}_{0} \mathbf{u}(t)+\sum_{i=1}^{N_{B}} \mathbf{B}_{i} \mathbf{u}\left(t-\eta_{i}\right) \\
& +\int_{0}^{L} \mathbf{A}(\tau) \mathbf{x}(t-\tau) \mathrm{d} \tau+\int_{0}^{L} \mathbf{B}(\tau) \mathbf{u}(t-\tau) \mathrm{d} \tau \\
\mathbf{y}(t) & =\mathbf{C} \mathbf{x}(t)
\end{aligned}
$$

Considering model (1) and zero initial conditions, the following input-output description of a general multi-input multi-output (MIMO) system in the form of the transfer matrix using the Laplace transform is obtained

$$
\begin{aligned}
\mathbf{Y}(s) & =\mathbf{G}(s) \mathbf{U}(s)=\frac{\mathbf{C a d j}[s \mathbf{I}-\mathbf{A}(s)] \mathbf{B}(s)}{\operatorname{det}[s \mathbf{I}-\mathbf{A}(s)]} \mathbf{U}(s) \\
\mathbf{A}(s) & =s \sum_{i=1}^{N_{H}} \mathbf{H}_{i} \exp \left(-s \eta_{i}\right)+\mathbf{A}_{0}+\sum_{i=1}^{N_{A}} \mathbf{A}_{i} \exp \left(-s \eta_{i}\right) \\
& +\int_{0}^{L} \tilde{\mathbf{A}}(\tau) \exp (-s \tau) \mathrm{d} \tau \\
\mathbf{B}(s) & =\mathbf{B}_{0}+\sum_{i=1}^{N_{B}} \mathbf{B}_{i} \exp \left(-s \eta_{i}\right)+\int_{0}^{L} \tilde{\mathbf{B}}(\tau) \exp (-s \tau) \mathrm{d} \tau
\end{aligned}
$$

All transfer functions in $\mathbf{G}(s)$ (or a transfer function in SISO case) have identical denominator factor in the form

$$
\begin{aligned}
m(s) & =\operatorname{numdet}[s \mathbf{I}-\mathbf{A}(s)]=\operatorname{num} M(s) \\
& =s^{n}+\sum_{i=0}^{n} \sum_{j=1}^{h_{i}} m_{i j} s^{i} \exp \left(-s \eta_{i j}\right), \eta_{i j} \geq 0
\end{aligned}
$$

where prefix num means the numerator of the determinant, and $\sum_{j=1}^{h_{n}} m_{n j} \exp \left(-\eta_{n j} s\right) \neq$ constantholds for a neutral system; otherwise, the system is retarded. The expression on the righthand side of (3) represents a so called quasipolynomial (El'sgol'ts \& Norkin, 1973). In the case of internal distributed delays, $M(s) \neq m(s)$ represents a meromorphic function and zeros of $m(s)$ do not agree with system poles. Moreover, inputoutput distributed delays bring about additional denominator factors as $\operatorname{den}(\operatorname{Cadj}[s \mathbf{I}-\mathbf{A}(s)] \mathbf{B}(s))$ for some transfer functions in $\mathbf{G}(s)$,

LTI-TDS is asymptotically stable if all poles are located in the open left half plane, $\square_{0}^{-}$. In the case of neutral systems, one has to check sensitivity to infinitesimal change in delays which can destabilize the system. In this sense, necessary and sufficient strong stability condition in the Laplace transform can be formulated as

$$
\sum_{j=1}^{h_{i}}\left|m_{n j}\right|<1
$$


see e.g. (Hale \& Verduyn Lunel, 1993; Zítek \& Vyhlídal, 2008) where $m_{n j}$ are coefficients for the highest $s$-power in (3). A strongly stable system is robust against infinitesimal changes in delays of a neutral LTI-TDS which can destroy the asymptotic stability of the difference equation. Strong stability implies formal stability which means that a neutral LTI-TDS has only a finite number of poles in the (closed) right-half complex plane, $\square^{+}$(Pontryagin, 1942; Loisseau et al., 2002).

Let $m(s)$ be a retarded or strongly stable neutral quasipolynomial. The number $N_{U}$ of its zeros $m(s)$ located in $\square^{+}$is

$$
N_{U}=\frac{n}{2}-\frac{1}{\pi} \underset{s=\mathrm{j} \omega, \omega \in[0, \infty)}{\arg m(s)}
$$

see e. g. (Górecki et al., 1989).

Consider quasipolynomial $m(s)$ of neutral type satisfying $m(0)>0, m(s) \neq 0$ for any imaginary $s=\mathrm{j} \omega, \omega \in \square$, and (4). Then $m(s)$ is strongly and asymptotically stable if and only if

$$
\frac{n \pi}{2}-\Phi \leq \underset{s=\mathrm{j} \omega, \omega \in[0, \infty)}{\arg m}(s) \leq \frac{n \pi}{2}+\Phi, \Phi=\arcsin \left(\sum_{j=1}^{h_{n}}\left|m_{n j}\right|\right)
$$

according to (Zítek \& Vyhlídal, 2008).

\section{NYQUIST CRITERION FOR LTI-TDS WITH DISTRIBUTED DELAYS}

Consider a simple control system as in Fig. 1 and express the plant and controller transfer functions, respectively, as

$$
G(s)=b(s) / a(s), G_{R}(s)=q(s) / p(s)
$$

where $a(s), b(s), q(s), p(s)$ are quasipolynomials and $G(s)$ is strictly proper and $G_{R}(s)$ is proper (the properness is defined as for delay-free systems using the highest $s$-power). The theorem reads as follow.

Let the plant and the controller have transfer functions as in (7) with distributed delays and let the control system be of the simple feedback structure. Let quasipolynomials $a(s)$ and $p(s)$ have no root on the imaginary axis, i.e. $a(s) \neq 0, p(s) \neq 0$ for any imaginary $s=\mathrm{j} \omega, \omega \in \square$, and the denominator of $G_{O}(s)$ be $m_{a p}(s)$. Then

1) If $m_{a p}(s)$ is a retarded quasipolynomial with

$$
\underset{s=\mathrm{j} \omega, \omega \in[0, \infty)}{\Delta \arg } m_{a p}(s)=l \pi / 2
$$

then the closed-loop system is asymptotically stable if and only if

$$
\underset{s=\omega j, \omega \in[0, \infty)}{\Delta \arg }\left(1+G_{O}(s)\right)=\left(n-l-2 \bar{n}_{U}\right) \frac{\pi}{2}=\bar{n}_{U, a p} \pi
$$

holds where $n$ is the highest $s$-power in $m_{a p}(s), \bar{n}_{U}$ stands for the number of common zeros of the numerator and denominator of $G_{O}(s)$ in $\square^{+}$and $\bar{n}_{U, a p}$ represents for the number of unstable zeros of $m_{a p}(s)$ which are not included in the numerator of $G_{O}(s)$.
2) If $m_{a p}(s)$ is a neutral quasipolynomial satisfying (4) then the closed-loop system is asymptotically and strongly stable if and only if (9) holds.

\section{CONCLUSION}

In this contribution, a generalized Nyquist criterion for both retarded and neutral LTI-TDS with distributed delays has been presented. Dealing with quasipolynomials instead of polynomials is a rather more involved since an infinite spectrum of such systems. Moreover, the presence of distributed delays makes the decision about system stability more complex because transfer function denominator does not coincidence with the system poles. Neutral systems require including a notion of strong stability as well.

In the future work, the presented theorem will be utilized while testing the robustness of controllers designed for LTITDS using the algebraic approach in the $\mathbf{R}_{M S}$ ring, see e.g. (Pekař \& Prokop, 2011).

\section{ACKNOWLEDGEMENTS}

The author kindly appreciate the financial support which was provided by the Ministry of Education, Youth and Sports of the Czech Republic, in the grant No. MSM 7088352102 and by the European Regional Development Fund under the project CEBIA-Tech No. CZ.1.05/2.1.00/03.0089.

\section{REFERENCES}

El'sgol'ts, L. E. \& Norkin, S. B. (1973). Introduction to the Theory and Application of Differential Equations with Deviated Arguments, Academic Press, New York

Górecki, H.; Fuksa, S.; Grabowski, P. \& Korytowski, A. (1989). Analysis and Synthesis of Time Delay Systems, John Wiley \& Sons, ISBN 978-0471276227, New York

Hale, J. K. and Verduyn Lunel, S. M. (1993). Introduction to Functional Differential Equations, Vol. 99 of Applied Mathematical Sciences, Springer-Verlag, ISBN 9780387940762, New York

Loiseau, J. J.; Cardelli, M \& Dusser, X (2002). Neutral-Type Time-Delay Systems that are not Formally Stable are not BIBO Stabilizable, IMA Journal of Math. Control and Information, Vol. 19, Issue 1-2, 217-227, ISSN 02650754

Pekař, L.; Matušů, R.; Dostálek, P. \& Dolinay J. (2011). The Nyquist Criterion for LTI Time-Delay Systems, Recent Researches in Automatic Control, May 27-29 2011, Lanzarote, Canary Islands, Spain, ISBN 978-1-61804-0046, pp. 80-86, WSEAS Press

Pekař, L. \& Prokop, R. (2011). On Reference Tracking and Disturbance Rejection for Time Delay Systems, Proceedings of the IASTED International Conference on Modelling, Identification and Control, February 15-17 2011, Innsbruck, Austria, ISBN 978-088986863-2, pp. 327333, Acta Press

Pontryagin, L. S. (1942). On the Zeros of Some Elementary Transcendental Functions. Izvestiya Akademii Nauk SSSR, Vol. 6, No. 33, 1942, pp. 115-131

Stépán, G. (1989). Retarded Dynamical Systems: Stability and Characteristic Functions, Vol. 210 of Pitman Research Notes in Mathematics Series, Longman Scientific and Technical, ISBN 978-0582039322, New York

Zítek, P. \& Vyhlídal, T. (2008). Argument-increment Based Stability Criterion for Neutral Time Delay Systems, Proceedings of the 16th Mediterranean Conference on Control and Automation, 25-27 June 2008, Ajaccio, France, ISBN 978-1-4244-2504-4, pp. 824-829 